\title{
EL CRECIMIENTO URBANO Y SU INFLUENCIA EN LOS CAUDALES DE CRECIDA: UN CASO DE ESTUDIO EN UNA CUENCA URBANA EN LOS ANDES ECUATORIANOS
}

\author{
URBAN GROWTH AND ITS INFLUENCE ON FLOOD FLOWS: A CASE \\ STUDY IN AN URBAN BASIN IN THE ECUADORIAN ANDES.
}

\author{
Fernando Oñate-Valdivieso ${ }^{1}$, Arianna Oñate-Paladines ${ }^{2}$ \\ ${ }^{1}$ Departamento de Geología, Minas e Ingeniería Civil. Universidad Técnica Particular de Loja. C/. Marcelino \\ Champagnat S/N 1101608. Loja Ecuador.Email: fronate@utpl.edu.ec \\ ${ }^{2}$ Euroaquae Master Program, Newcastle University, King's Gate Newcastle Upon Tyne NE1 7RU, Newcastle, \\ $U K$.
}

Recibido: 08 de agosto 2019 / Aceptado: 09 de diciembre 2019

\begin{abstract}
RESUMEN
El notable crecimiento urbano que han experimentado varias ciudades en el Ecuador en los últimos años ha provocado cambios en el uso de suelo de las cuencas hidrográficas de las que ellas forman parte. Desde el punto de vista hidrológico el cambio de uso del suelo se ve reflejado en el cambio de la respuesta de una cuenca hidrográfica a eventos extremos de precipitación. En el presente estudio se realiza un análisis multitemporal (1976- 2010) del crecimiento urbano de la ciudad de Loja, capital de la provincia ecuatoriana del mismo nombre, determinándose el crecimiento experimentado por la ciudad y la variación del uso de suelo en la cuenca del río Zamora, de la que Loja es parte, durante el período considerando. Para la evaluación de los caudales de crecida, según las diferentes etapas de crecimiento urbano, se implementó un modelo hidrológico de evento. El análisis multitemporal del crecimiento urbano, la obtención de parámetros hidrológicos y la presentación de resultados se realizó aplicando Sistemas de Información Geográfica. Los resultados permiten demostrar la relación directa entre el crecimiento del área urbana y el incremento de la magnitud de los caudales de crecida.
\end{abstract}

Palabras clave: Hidrología urbana, crecimiento superficie urbana, modelo hidrológico, caudales de crecida

\begin{abstract}
The remarkable urban growth that several cities in Ecuador have experienced in recent years, has led to changes in the land use of the river basins of which they are part. From a hydrological point of view, the change in land use is reflected in the change in the response of a river basin to extreme precipitation events. In this study, a multitemporal analysis (1976-2010) of the urban growth of the city of Loja, capital of the Ecuadorian province of the same name, is performed, determining the growth experienced by the city and the variation of land use in the basin of the
\end{abstract}


Zamora river, of which Loja is a part, during the period under consideration. For the evaluation of flood flows, according to the different stages of urban growth, an event hydrological model was implemented. The multitemporal analysis of urban growth, the obtaining of hydrological parameters and the presentation of results was carried out by applying Geographic Information Systems. The results show the direct relationship between the growth of the urban area and the increase in the magnitude of flood flows.

Keywords: Urban hydrology, urban surface growth, hydrological model, flood flows

\section{INTRODUCCIÓN}

En la actualidad existe un creciente interés en conocer cómo las actividades humanas influyen en las distintas variables climáticas e hidrológicas. Varios estudios han reportado cambios significativos ocasionados por la influencia humana en variables climáticas como la temperatura y la precipitación (Hegerl et al., 1996; Zhang et al., 2007; Santer et al., 2011), o en variables hidrológicas como el caudal (Chelsea Nagy et al., 2012; Huang et al., 2012; Cruise et al., 2010; Zheng et al., 2012).

Entre las actividades humanas que afectan las variables hidrológicas se destacan la urbanización, los cambios en las prácticas agrícolas y la construcción de las estructuras hidráulicas, entre otras. Varios estudios han analizado el efecto de la urbanización (Chelsea Nagy et al., 2012 y Huang et al., 2012) o el cambio de uso de la tierra sobre la hidrología (Cruise et al., 2010; y Zheng et al., 2012; Oñate-Valdivieso y Bosque, 2014).

El proceso de urbanización tiene impactos significativos en la hidrología de una cuenca hidrográfica, ocasionando una disminución de la infiltración y del flujo base, así como un aumento de los caudales de crecida y los volúmenes de escorrentía. El proceso de urbanización crea áreas impermeables y los sistemas de drenaje pluvial simplifican el sistema de drenaje natural alterando la respuesta de la cuenca ante eventos de precipitación ya que se producen tiempos de concentración más cortos así como la reducción de los tiempos de recesión (Chow et al., 1994; Fletcher et al., 2012).

La urbanización es una tendencia a nivel mundial. Actualmente más del $50 \%$ de la población del mundo vive en centros urbanos, existiendo hoy en día más de 500 ciudades con una población que supera el 1 millón de habitantes (Fletcher et al., 2012; United Nations, 2010). Las razones del crecimiento son diversas; en el caso de las ciudades latinoamericanas se pueden citar al crecimiento demográfico natural, la migración del campo a la ciudad en busca de mejores condiciones de vida y cambios en los patrones de localización de actividades económicas y de vivienda, entre otros.

Varias ciudades en el Ecuador han experimentado un acelerado crecimiento, que se pone de manifiesto en un notable incremento del área urbanizada en los últimos años. Una de esas ciudades es Loja, capital de la provincia del mismo nombre, ubicada al sur de la República del Ecuador y fronteriza con el Perú. En el presente estudio se analiza la influencia que este crecimiento ha tenido en la hidrología de la cuenca y en los eventos extremos de caudal que en ella se producen; para ello se realizó el análisis multitemporal de la variación de superficie urbana a partir de fotografías aéreas e imágenes de satélite, para luego, empleando datos de precipitación y aplicando un modelo hidrológico, generar caudales de crecida para diversos escenarios de cobertura y evaluar el efecto que dichos caudales tendrían en las zonas aledañas a las riveras de los ríos en varios lugares de interés. El procesamiento de la información espacial se realizaró aplicando Sistemas de Información Geográfica (SIG). 


\section{MATERIALES Y MÉTODOS}

\section{ÁREA DE ESTUDIO}

El presente estudio se realiza en la ciudad de Loja, ubicada al sur del Ecuador a $79.21^{\circ} \mathrm{de}$ longitud oeste y $3.99^{\circ}$ de latitud sur (Municipio de Loja, 2014), en un valle interandino. La ciudad tiene una extensión aproximada de 2993.99 Ha y cuenta con una población cercana a los 200000 habitantes (Municipio de Loja, 2014). La ciudad ocupa la parte media de la cuenca inicial del río Zamora (22 $747.50 \mathrm{Ha}$ ). El río Zamora presenta períodos de estiaje entre los meses de mayo a noviembre, pudiendo presentar significativos caudales de crecida en la temporada lluviosa. La cuenca posee una altura media de $2400 \mathrm{msnm}$, una pendiente media del $30 \%$, la pendiente media del cauce principal es de 8.3\% (Carvajal y Piedra, 2007). La cuenca posee cobertura vegetal en buena condición pudiendo identificarse principalmente pastizales, matorrales y cobertura boscosa. La ubicación del área de estudio se presenta en la Figura 1.
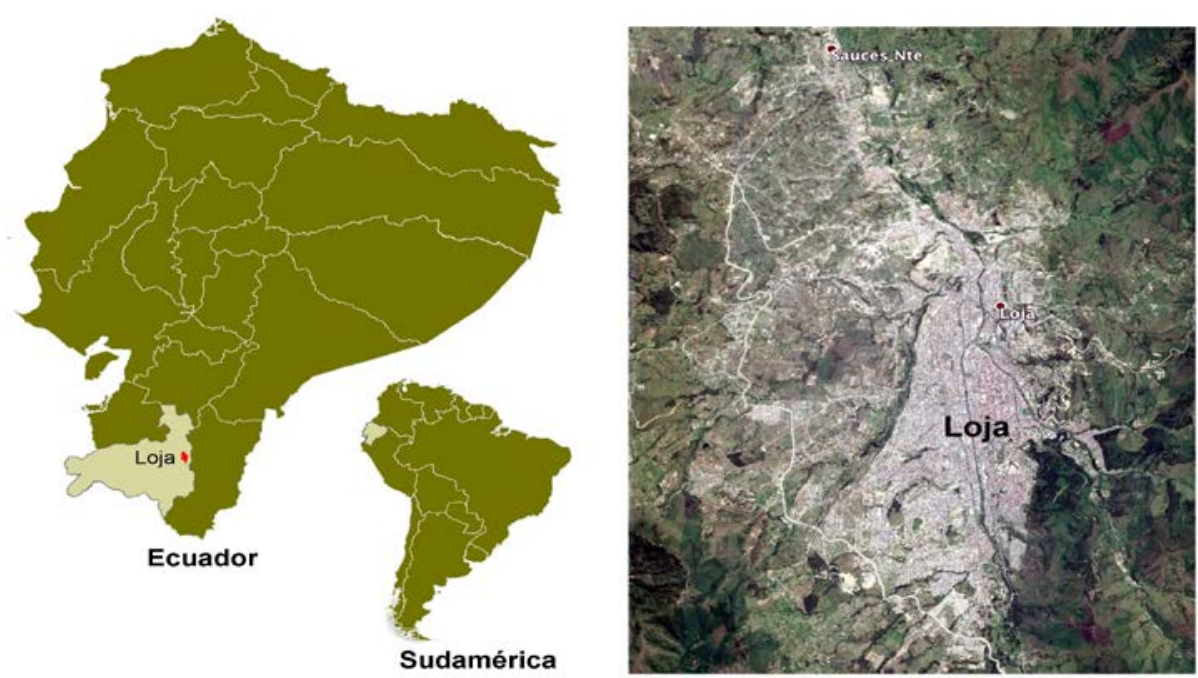

Figura 1. Ubicación de la zona de estudio (Google, 2019)

\section{ANÁLISIS MULTITEMPORAL DEL CRECIMIENTO URBANO Y GENERACIÓN DE ESCENARIOS}

El análisis multitemporal del crecimiento de la superficie urbana se realizó a partir de la fotointerpretación de fotografías aéreas correspondientes a los años 1976, 1984, 1998 (Esc. 1:50 000) (IGM, 2018), 2002 (Municipio de Loja, 2003), 2010 (MAG, 2019), georeferenciadas, obteniéndose los polígonos correspondientes al área urbana en cada una de esas fechas mediante fotointerpretación. Se recopiló información histórica de tipo y uso de suelo de la zona de estudio (MAG, 2019), la que combinada con los datos del crecimiento urbano de la ciudad, permitió elaborar mapas particulares para cada variable y para cada año considerado, diez en total,

Obtenidos los mapas de ocupación del suelo se optó por analizar los cambios ocurridos entre 1984 y 1998 relacionándolos con las posibles variables explicativas y obtener un modelo predictivo que sería validado mediante comparación con la cobertura obtenida para 2002. Los cambios ocurridos, se estudiaron aplicando la metodología propuesta por Pontius et al. (2004), la que permite determinar la persistencia, ganancia, pérdida e intercambios entre las categorías temáticas consideradas en cada mapa de ocupación del suelo mediante el análisis de una tabulación cruzada de ellos, identificándose las transiciones ocurridos entre 1984, 1998. Las 
relaciones entre las transiciones observadas y sus posibles variables explicativas se denominan submodelos de transición. El número de submodelos de transición será igual al número de transiciones que se produzcan en la zona de estudio; siendo posible agrupar varias transiciones en un solo modelo, cuando se considera que éstas son producto de las mismas causas.

Cada modelo de transición incluye un cierto número de variables explicativas, las que pueden ser seleccionadas en base a su potencial explicativo, calculado por el coeficiente $V$ de Cramer, o ensayando varias combinaciones de variables explicativas, hasta obtener el ajuste óptimo entre transiciones y variables explicativas. Valores de $V$ de Cramer mayores a 0.4 son aceptables (Eastman, 2006). Tres variables explicativas fueron consideradas: La elevación (empleando un modelo de elevación digital - DEM), que determina la presencia de diferentes tipos de vegetación; La pendiente del terreno que limita el crecimiento urbano; y, la distancia a calles y vías, que motiva y facilita el crecimiento urbano.

Los submodelos de transición se calcularon mediante regresión logística y mediante una red neuronal de perceptrones multicapa $(M L P)$, obteniéndose la probabilidad de ocurrencia de cada transición según las variables explicativas seleccionadas. La regresión logística (Kleinbaum y Klein, 2002, p. 4) permite establecer una relación entre una variable dependiente binaria (transiciones) y las variables explicativas consideradas, modelando su probabilidad de ocurrencia según éstas.

Las redes neuronales de perceptrones multicapa por su parte están formadas por un conjunto de elementos simples (neuronas o perceptrones) distribuidos en capas y que están conectadas con la capa o capas intermedias mediante funciones de activación. Esas funciones se definen a partir de una serie de pesos o factores de ponderción que se calculan de modo interactivo en el proceso de aprendizaje de la red. El objetivo de dicho aprendizaje es estimar unos resultados conocidos (transiciones observadas) a partir de unos datos de entrada (variables explicativas); para posteriormente, calcular resultados desconocidos a partir del resto de los datos de entrada. El aprendizaje se realiza a partir de todas las unidades que forman la red, variando el conjunto de los pesos en interacciones sucesivas (Chuvieco, 2010; Pijanowski et al., 2002).

El modelamiento del cambio de ocupación del suelo hacia un año horizonte se realizó aplicando cadenas de Markov. En el presente caso utilizando el mapa de cobertura de la fecha final (1998) conjuntamente con la fecha a la que se quiere predecir el cambio de ocupación del suelo (2002), y la matriz de probabilidad de transición ya calculada, se determinan las zonas que experimentarán una transición desde la fecha final hasta la fecha de predicción.

El mapa de ocupación del suelo futuro fue modelado mediante un procedimiento de asignación multiobjetivo de usos de suelo (MOLA) (Oñate-Valdivieso y Bosque, 2010; Eastman, 2006). Considerando todas las transiciones y empleando las variables explicativas seleccionadas; se crea una lista de clases anfitrionas (que perderían alguna porción de terreno) y una lista de clases demandantes (que ganarían terreno).

Las áreas de pérdida o ganancia se determinan mediante cadenas de Markov; $\mathrm{y}$, mediante el procedimiento de asignación mutiobjetivo, en el que las variables explicativas determinan los lugares más adecuados para cada cambio de ocupación, se asigna terreno de todas las clases anfitrionas a todas las clases demandantes. Los resultados de cada reasignación de ocupación del suelo son superpuestos para producir el resultado final (Eastman, 2006). Información adicional puede encontrarse en Oñate-Valdivieso y Bosque (2010)

Con el procedimiento descrito, se generaron dos mapas que pronostican la ocupación del suelo para el año 2010 en base al modelamiento de las relaciones entre los cambios observados y las variables explicativas, dichas relaciones se modelaron con regresión logística y con redes neuronales.

Para la validación se consideró como referencia al mapa extraído de la imagen de 2001 y mediante matrices de confusión de estudió la correspondencia entre el mapa de referencia y 
los obtenidos mediante redes neuronales y regresión logística, pudiendo determinar los errores de pronóstico de las ocupaciones del suelo según cada modelo planteado, así como los errores de omisión y comisión que se hubiesen producido. De la matriz de confusión de calculó la fiabilidad global de la clasificación como la relación entre el número de píxeles correctamente asignados y el número total de píxeles de la imagen (Chuvieco, 2002,).Complementariamente se calculó el ajuste entre el mapa de referencia y los mapas generados mediante el índice Kappa (Pontius et al. 2004).

Analizado el ajuste, se procedió a generar un mapa de ocupación del suelo hacia el año 2020, considerando para ello los mapas de ocupación del suelo de 2002 y 2010, las variables explicativas seleccionadas para cada transición y aplicando el modelo que presente las mejores capacidades.

\section{MODELIZACIÓN HIDROLÓGICA}

Se creó un modelo hidrológico de evento aislado para el estudiar la respuesta de la cuenca del río Zamora ante eventos extremos de precipitación considerando además, las diferentes etapas de crecimiento urbano de la ciudad de Loja.

La topología de la cuenca fue elaborada en función de un modelo de elevación digital generado empleando un mapa de curvas de nivel a escala 1:50 000 (IGM, 1990). Este modelo topológico incluyó subcuencas aportantes, puntos de unión en los que se suman las aportaciones de las subcuencas, tramos de red fluvial en las que se realiza el tránsito hidrológico de los hidrogramas y el punto de salida de la cuenca en el que se obtiene el caudal resultante de la simulación lluvia-escorrentía. El modelo topológico se presenta en la Figura 2.

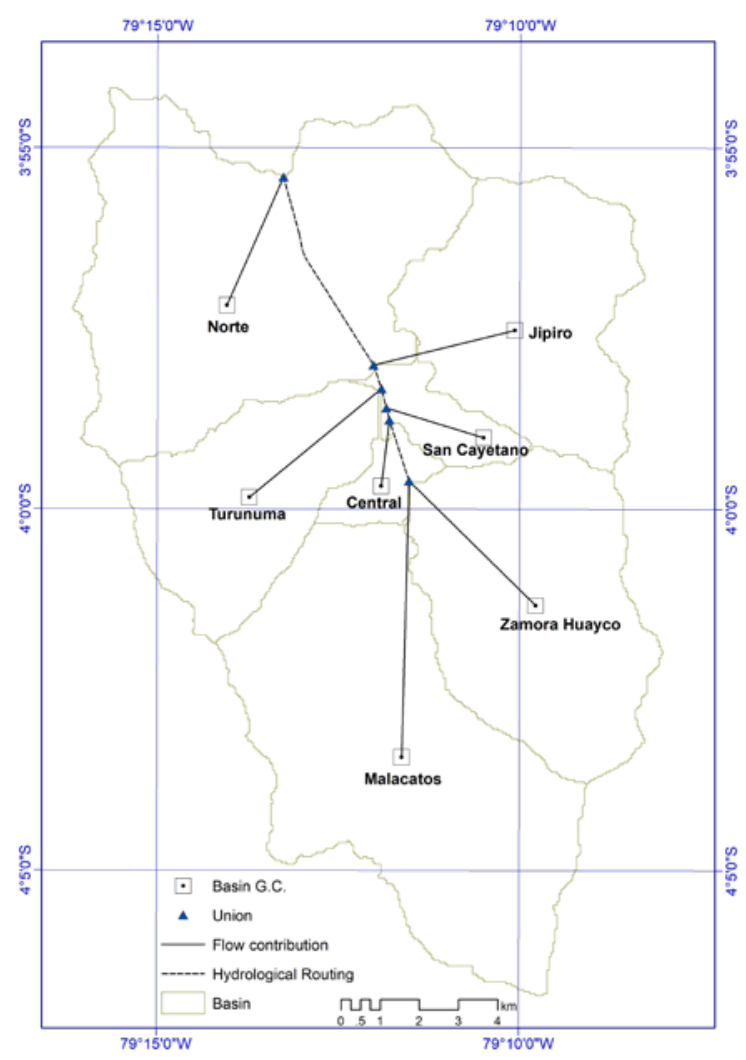

Figura 2. Modelo topológico de la cuenca del río Zamora 
Se generaron tormentas sintéticas para períodos de retorno de 10, 25, 50 y 100 años empleando ecuaciones de intensidad determinadas para la ciudad de Loja (INAMHI, 2019).

$$
\begin{gathered}
I_{T R}=92.854 I d_{T R} t^{-0.4083} \\
I_{T R}=480.74 I d_{T R} t^{-0.8489}
\end{gathered}
$$

En donde $I d_{T R}$ es la intensidad máxima para un período de retorno determinado, $t$ es la duración de la tormenta en minutos, $I_{T R}$ es la intensidad en $\mathrm{mmh}^{-1}$. La ecuación 1 es válida para duraciones entre 5 y 43 minutos, la ecuación 2 es válida para duraciones entre 43 minutos y 1440 minutos.

Las abstracciones se cuantificaron aplicando la metodología del número de la curva (CN) del US Soil Conservation Service (USSCS) (Dingman, 2015; Chow et al., 1994) para condiciones normales de humedad, calculándose el CN para cada una de las distintas unidades de respuesta hidrológica obtenidas según la intersección de la cartografía de uso y tipo de suelo para cada fecha considerada.

La transformación de escorrentía superficial en caudal se realizó aplicando el Hidrograma Unitario del USSCS. Para el tránsito hidrológico de caudales el método de Muskingum y Cunge fue aplicado. Los tiempos de concentración y de retraso de cada una de las microcuencas se determinaron aplicando la fórmula de Kirpich (Dingman, 2015; Chow et al., 1994).

\section{ANÁLISIS DE RESULTADOS}

El crecimiento urbano de la ciudad de Loja entre 1976 y 2010 se observan en la Figura 3 y un resumen del área ocupada por la ciudad y su población respectiva en cada instante analizado se incluyen en la Tabla 1.

Tabla 1. Variación de la superficie urbana y de población en la ciudad de Loja. Período 1976-2010

\begin{tabular}{rrrrr}
\multicolumn{1}{c}{ Año } & Área Urbana (ha) & Población & $\begin{array}{c}\text { Incremento área } \\
\text { urbana (\%) }\end{array}$ & $\begin{array}{c}\text { Crecimiento medio } \\
\text { anual de área urbana } \\
\text { (\%) }\end{array}$ \\
\hline 1976 & 606.02 & 47697 & 79.0 & 9.88 \\
1984 & 1084.86 & 71652 & 69.4 & 4.96 \\
1998 & 1838.18 & 107130 & 4.2 & 1.05 \\
2002 & 1915.31 & 118532 & 56.3 & 7.04 \\
2010 & 2993.99 & 185321 & & \\
\hline
\end{tabular}




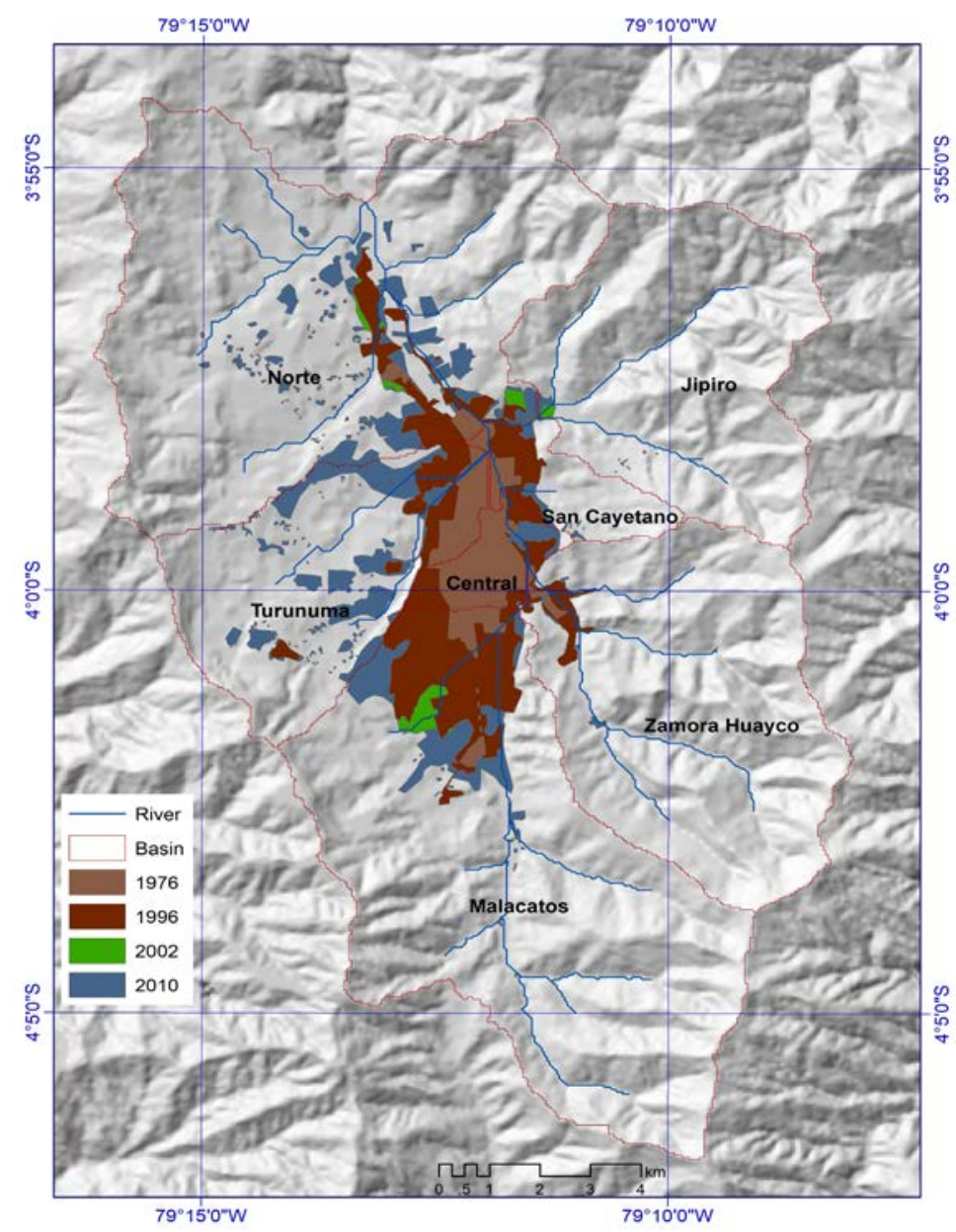

Figura 3. Evolución de la superficie urbana de la ciudad de Loja. Período: 1976-2010

$\mathrm{Al}$ analizar los datos incluidos en la Tabla 1 se observa que el crecimiento de la superficie urbana no presenta un patrón definido, ya que si se observa el crecimiento medio anual de la superficie urbana los valores fluctúan entre 1.05 \% y 9.88 \%. Esta variación del crecimiento medio anual podría tener relación con eventos importantes de la historia reciente de Ecuador, entre los que se puede citar (1) La significativa migración interna ocurrida en la década de 1970, especialmente desde zonas rurales de la provincia de Loja hacia centros urbanos, originada por un largo período de sequía extrema; (2) La recesión económica de 2000 que desaceleró el crecimiento económico del país y (3) La gran ola de migratoria hacia Europa y USA en la década del 2000. La búsqueda de relación entre estas y otras variables, excede el alcance del presente trabajo, pero debería investigarse con mayor profundidad.

\section{DETECCIÓN DE CAMBIOS}

La Tabla 2 presenta el resumen de la tabulación cruzada de datos para el período comprendido entre 1984 y 1996. Como se puede observar, existe un predominio de persistencia en todas las cubiertas. Hay 62,519.00 Ha de áreas estables, equivalentes al 98.77\% del área total de estudio, y 778.00 Ha de zonas que han experimentado cambios, correspondientes al 1.23\% del área total. Hay un aumento en las áreas urbanas y, en consecuencia, una disminución en las áreas rurales que estaban ocupadas antes de que ocurriera la expansión urbana. 
Tabla 2. Tabulación cruzada de los usos del suelos entre 1984 (columnas) and 1996 (filas)

\begin{tabular}{lrrr}
\hline & Country & \multicolumn{1}{c}{ City } & \multicolumn{1}{c}{ Total } \\
\hline Country & 61416.25 & 0.00 & 61416.25 \\
City & 778.00 & 1102.75 & 1880.75 \\
& & & \\
Total & 62194.25 & 1102.75 & 63297.00 \\
\hline
\end{tabular}

\section{VARIABLES EXPLICATIVAS}

La Tabla 3 muestra el grado de asociación entre las variables explicativas y las cubiertas de tierra presentes en el área de estudio. El valor de V de Cramer fluctúa entre 0.1 y 0.3. La pendiente es la variable que tiene la mayor asociación con las categorías de uso del suelo existentes (Tabla 3); Esto se debe a que la pendiente afecta la expansión urbana, así como los usos del suelo de las zonas rurales, como los cultivos o la presencia de bosques naturales. Otro nivel importante de asociación es el que se encuentra entre la elevación (DEM) y la presencia de la cobertura del suelo (Tabla 3), lo que demuestra que la elevación condiciona la expansión urbana.

Tabla 3. Valores de la V de Creamer: nivel de asociación entre variables explicativas cuantitativas y usos de suelo estudiados.

\begin{tabular}{lrr}
\hline & Área Rural & Área Urbana \\
\hline DEM & 0.3832 & 0.3832 \\
Pendiente & 0.4387 & 0.4387 \\
Distancia a vías & 0.3184 & 0.3184 \\
Distancia a ríos & 0.0367 & 0.0367 \\
\hline
\end{tabular}

La distancia a las carreteras tiene una V de Cramér aceptable, corroborando la suposición inicial de que la presencia de carreteras fomenta la expansión urbana. La distancia a los ríos tiene valores de $\mathrm{V}$ de Cramér $<0.1$, probablemente porque no hay una regulación estricta de la expansión urbana en áreas cercanas a los ríos.

\section{SUBMODELOS DE TRANSICIÓN}

La Tabla 4 muestra los diferentes submodelos de transición con sus respectivas variables y los resultados de la regresión logística calculada. Se incluyen los coeficientes que afectan cada variable explicativa en la ecuación de regresión logística y la correlación entre variables y transiciones (ROC).

Tabla 4. Resultados de regresión logística: transición modelada (submodelo de transición), correlación (ROC), variables explicativas y coeficientes de cada variable explicativa en la ecuación de regresión.

\begin{tabular}{cllr}
\hline Transición & ROC & \multicolumn{1}{c}{ Variables } & Coeficiente \\
\hline \multirow{2}{*}{ De rural a urbano } & \multirow{2}{*}{0.9508} & Intercept & 7.8911 \\
& & DEM & -0.0032 \\
& Pendiente & -0.1251 \\
& & Distancia a vías & -0.5805 \\
\hline
\end{tabular}


La Tabla 4 muestra la relación inversa entre la transición del campo a la ciudad y todas las variables consideradas en el modelo de transición. Esto se debe al hecho de que las posibilidades de expansión urbana se reducen cuando hay una mayor elevación, así como un terreno más empinado y distancias más largas a las carreteras. El grado de correlación entre la transición estudiada y las variables explicativas es alto, alrededor del 95\%.

La Tabla 5 muestra los resultados de la aplicación de redes neuronales. La tasa de aprendizaje es baja, aproximadamente $1 / 1000$, con un error de capacitación y validación de alrededor de 2/10, que está muy por encima del error aceptable (RMS). Esto demuestra el rendimiento limitado de las redes neuronales en el presente caso, a pesar de que la tasa de precisión es superior al 90\%.

Tabla 5. Resultados de la aplicación de redes neuronales

\begin{tabular}{lr}
\hline Parámetro & Valor \\
\hline Capa de entrada neuronas & 3 \\
Capa oculta neuronas & 2 \\
Capa de salida neuronas & 2 \\
Muestras solicitadas por clase & 3112 \\
Tasa de aprendizaje final & 0.0003 \\
Factor de impulso & 0.5 \\
Constante sigmoidea & 1 \\
RMS aceptable & 0.01 \\
Iterations & 10000 \\
Entrenamiento RMS & 0.2595 \\
Prueba RMS & 0.2651 \\
Tasa de precisión & $91.23 \%$ \\
Medida de habilidad & 0.8245 \\
\hline
\end{tabular}

Las probabilidades de transición calculadas para las ocupaciones consideradas se incluyen en la Tabla 6, que muestra que las probabilidades de mantener el mismo uso del suelo predominan en otras transiciones posibles, alcanzando casi la unidad en el caso del campo, y con valores $>1$ en el caso del ciudad. Como se esperaba, no es probable que el campo cambie a una ciudad, mientras que la ciudad es siempre la misma.

Tabla 6. Probabilidad de transición entre usos del suelo

\begin{tabular}{lcc}
\hline & Área Rural & Área Urbana \\
\hline Área Rural & 0.9937 & 0.0063 \\
Área Urbana & 0 & 1 \\
\hline
\end{tabular}

La Tabla 7 muestra la matriz de confusión entre el mapa extraído de la imagen de 2002 y el mapa generado por las redes neuronales (MLP). La Tabla 8 muestra la matriz de confusión entre el mapa extraído de la imagen de 2002 y el mapa generado por regresión logística (LogReg). En ambas tablas, la comparación de los mapas muestra un predominio en el número de píxeles que tienen la misma clase temática. Los mayores errores ocurren cuando el área rural ha sido modeladas como área urbana (1497 y 1493 píxeles). Los errores en los que el área urbana sido modelada como área rural son menores (289 y 285 píxeles). Del mismo modo, los 
errores de comisión varían entre $0.61 \%$ y 3.68\%. El valor máximo corresponde a la ciudad en ambas tablas. Los errores de omisión varían entre $0.12 \%$ y $16.51 \%$, siendo el mayor error por comisión en el cambio a área urbana.

Tabla 7. Matriz de confusión entre el mapa extraído de la imagen de 2002 y el mapa creado a través de redes neuronales (MLP).

\begin{tabular}{lrrrr}
\hline & \multicolumn{2}{c}{ Mapa de 2002 (Referencia) } & & \multicolumn{1}{c}{$\begin{array}{c}\text { Error por } \\
\text { comisión (\%) }\end{array}$} \\
& Área Rural & Área Urbana & Total & \\
Map 2002 (MLP) & & & & \\
Área Rural & 243834 & 1497 & 245331 & 3.68 \\
Área Urbana & 289 & 7568 & 7857 & \\
& & & & \\
Total & 244123 & 9065 & 253188 & \\
Error por omisión (\%) & 0.12 & 16.51 & & \\
\hline
\end{tabular}

Tabla 8. Matriz de confusión entre el mapa extraído de la imagen de 2002 y el mapa creado mediante regresión logística (LogReg)

\begin{tabular}{lrrrr}
\hline & \multicolumn{2}{c}{ Mapa de 2002 (Referencia) } & & \multicolumn{1}{c}{$\begin{array}{c}\text { Error por } \\
\text { comisión (\%) }\end{array}$} \\
\hline Map 2002 (Reg-Log) & Área Rural & Área Urbana & \multicolumn{1}{c}{ Total } & \multicolumn{1}{c}{} \\
Área Rural & & & & \\
Área Urbana & 243838 & 7493 & 245331 & 0.61 \\
& 285 & 7572 & 7857 & 3.63 \\
Total & & & & \\
Error por omisión (\%) & 244123 & 9065 & 253188 & \\
\hline
\end{tabular}

La Tabla 9 muestra los valores de la confiabilidad general calculada a partir de las matrices de confusión incluidas en las Tablas 7 y 8, así como el índice de Kappa y el coeficiente de correlación entre el mapa de referencia de 2002 y los mapas de uso del suelo generados con regresión logística y redes neuronales. El mapa generado por regresión logística tiene una confiabilidad total de $99.30 \%$, un índice Kappa de 0.8913 y un coeficiente de correlación de 0.8938. Estos valores son más altos que los obtenidos usando redes neuronales, por un margen muy estrecho.

Tabla 9: Parámetros de validación entre el mapa extraído de la imagen de 2002 y los mapas creados mediante regresión logística (LogReg) y redes neuronales (MLP).

\begin{tabular}{lrr}
\hline & 2002 MLP & 2002 Reg-log \\
\hline Confiabilidad general (\%) & 99.29 & 99.30 \\
Kappa & 0.8908 & 0.8913 \\
$\mathrm{R}$ & 0.8933 & 0.8938 \\
\hline
\end{tabular}




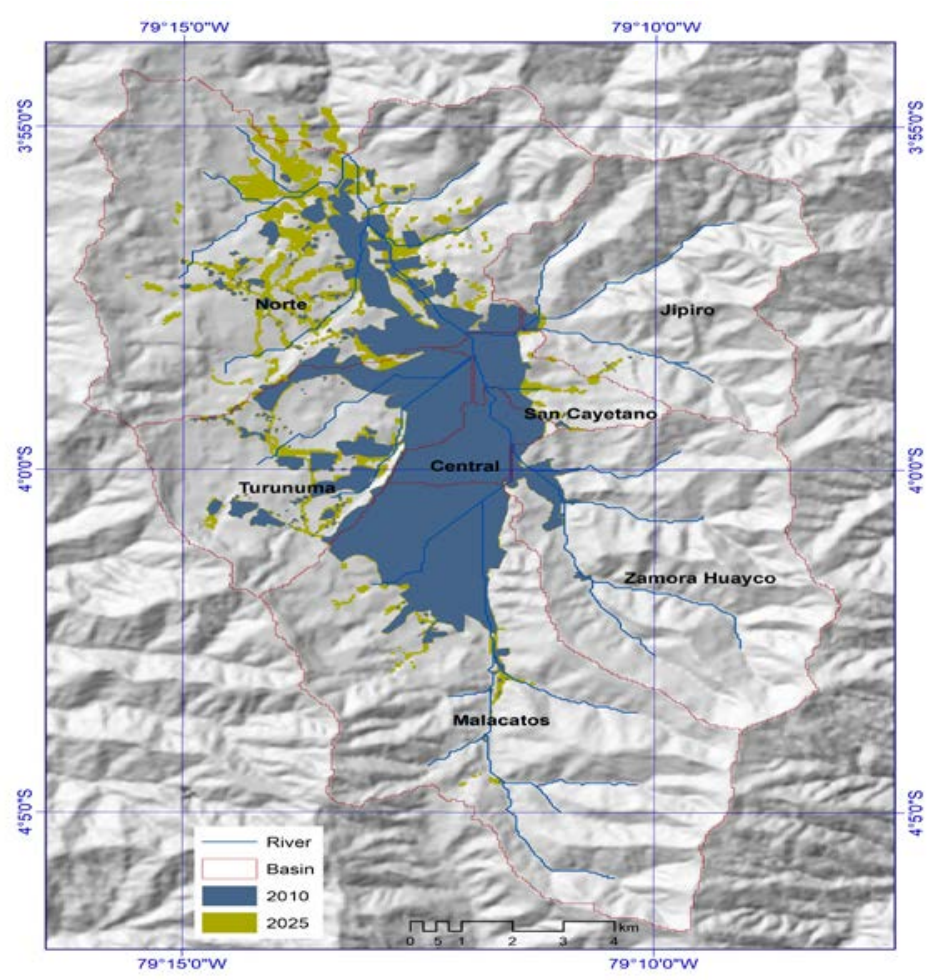

Figura 4. Escenario de crecimiento urbano para 2025, en comparación con la extensión de la ciudad en 2010

\section{MODELIZACIÓN HIDROLÓGICA}

Las características morfológicas de las microcuencas se presentan en la tabla 10, en esta se puede observar que los parámetros de infiltración (CN) sufren un incremento conforme incrementa la superficie urbana en cada microcuenca. Dicho incremento es relativamente reducido ya que por ejemplo en la subcuenca Malacatos, que es la que presenta la mayor variación del CN, alcanza un valor orden del 3\%. Esta pequeña variación se debe a que la superficie urbana en cada una de las subcuencas es relativamente pequeña si se compara con su superficie total. Las subcuencas que presentan mayor superficie urbana (véase figura 3) presentan un valor mayor del número de la curva.

El tiempo de concentración es relativamente corto y está relacionado con la pendiente del cauce principal la distancia máxima que debe recorrer la escorrentía.

Tabla 10. Características de las subcuencas en estudio

\begin{tabular}{lcccccccr}
\hline & \multicolumn{9}{c}{ Curve Number (CN) } & \multicolumn{2}{c}{ Area } \\
Subcuenca & $\mathbf{1 9 7 6}$ & $\mathbf{1 9 8 4}$ & $\mathbf{1 9 9 6}$ & $\mathbf{2 0 0 2}$ & $\mathbf{2 0 1 0}$ & (ha) & tc (h) & tlag (h) \\
\cline { 2 - 7 } Central & 93.4 & 95.0 & 95.0 & 95.0 & 95 & 422.00 & 0.44 & 0.27 \\
Jipiro & 65.1 & 65.1 & 65.3 & 65.4 & 66.2 & 3192.50 & 0.83 & 0.50 \\
Malacatos & 74.8 & 75.3 & 75.8 & 75.9 & 77.1 & 6027.50 & 1.64 & 0.99 \\
Norte & 76.1 & 76.2 & 76.8 & 76.9 & 77.4 & 6256.75 & 1.82 & 1.09 \\
San Cayetano & 74.3 & 75.5 & 77.3 & 77.3 & 77.3 & 580.25 & 0.47 & 0.28 \\
Turunuma & 74.4 & 74.4 & 74.9 & 75.0 & 76.3 & 2415.25 & 0.90 & 0.54 \\
Zamora Huayco & 65.6 & 65.8 & 66.0 & 66.0 & 66.5 & 3853.25 & 1.04 & 0.62 \\
\hline
\end{tabular}


El tiempo de retraso es relativamente corto y está relacionado con la pendiente del canal principal y la distancia máxima recorrida por la escorrentía.

Los valores de precipitación para diferentes duraciones y períodos de retorno se indican en la Tabla 11. Como se esperaba, los valores de precipitación aumentan a medida que aumenta el período de retorno y la duración.

Tabla 11. Valores de precipitación según el período de retorno.

\begin{tabular}{rrrrr}
\hline & \multicolumn{4}{c}{ Períodos de retorno (Años) } \\
\cline { 2 - 5 } Duración (min) & $\mathbf{1 0}$ & $\mathbf{2 5}$ & $\mathbf{5 0}$ & $\mathbf{1 0 0}$ \\
\cline { 2 - 5 } & \multicolumn{4}{c}{ Precipitación (mm) } \\
\hline 5 & 11 & 12 & 12.8 & 15.2 \\
15 & 21.1 & 23 & 24.6 & 29.2 \\
60 & 40.9 & 44.6 & 47.6 & 56.5 \\
120 & 45.4 & 49.5 & 52.8 & 62.7 \\
180 & 48.3 & 52.7 & 56.2 & 66.7 \\
\hline
\end{tabular}

Las tormentas incluidas en la tabla 11 aplicadas de manera individual según el período de retorno y el estado de crecimiento del área urbana de la ciudad de Loja permitieron obtener los caudales que se incluyen en la Tabla 12. Se puede apreciar una relación directa entre el período de retorno y los caudales de crecida, al igual que una relación directa entre el crecimiento del área urbana y los caudales de crecida para un mismo período de retorno. Para un mismo período de retorno se puede observar incrementos de caudales relacionados con incrementos de la superficie urbana.

Tabla 12. Caudales de crecida $\left(\mathrm{m}^{3} / \mathrm{s}\right)$ según instante considerado y período de retorno

\begin{tabular}{rrrrrr}
\hline & Area urbana & \multicolumn{5}{c}{ Período de retorno } \\
\cline { 3 - 6 } Año & (ha) & $\mathbf{1 0}$ & $\mathbf{2 5}$ & $\mathbf{5 0}$ & $\mathbf{1 0 0}$ \\
\hline 1976 & 606.02 & 95.3 & 136.50 & 169.30 & 282.80 \\
1984 & 1084.86 & 100.3 & 142.40 & 175.60 & 290.90 \\
1996 & 1838.18 & 105.9 & 148.80 & 182.90 & 300.90 \\
2002 & 1915.31 & 107.9 & 151.00 & 185.60 & 304.50 \\
2010 & 2993.99 & 113.3 & 162.70 & 194.45 & 319.63 \\
2025 & 3932.50 & 140.3 & 178.12 & 208.57 & 328.71 \\
\hline
\end{tabular}

La relación entre el caudal, los períodos de retorno y el crecimiento urbano se presenta en la figura No. 5 en esta se puede apreciar una alta correlación entre la superficie urbana y la magnitud de los caudales observándose esta correspondencia en los distintos períodos de retorno considerados. 


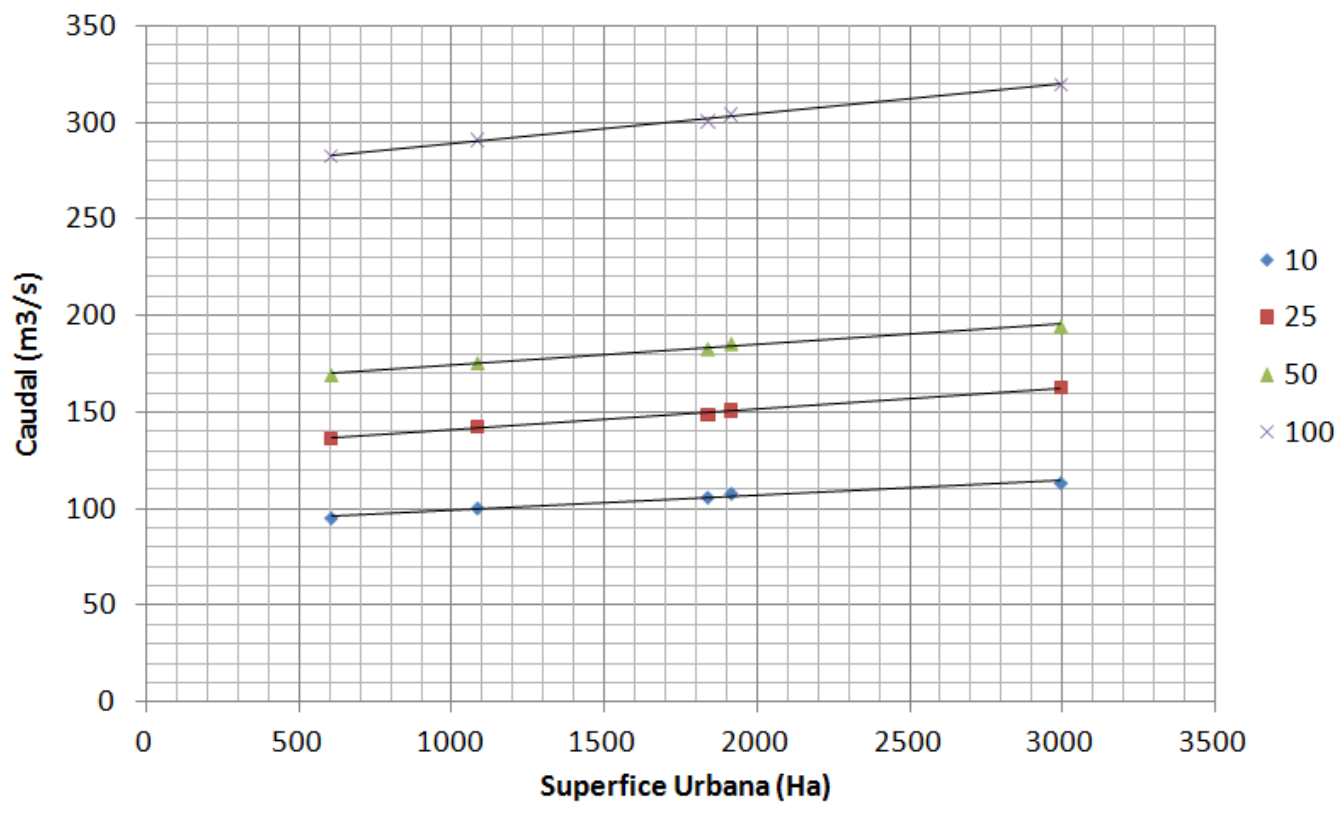

Figura 5. Relación entre el caudal de crecida, los períodos de retorno y el crecimiento urbano de la ciudad de Loja.

Durante el período de estudio, el área urbana de la ciudad de Loja experimentó un aumento considerable, pasando de 606.02 Нa a 2993.99 Ha, un aumento equivalente de 394.04\%. El área total de la cuenca del río Zamora es de 22,747.50 Ha, por lo que en 2010 la ciudad de Loja cubriría solo el $13.16 \%$ de la cuenca total, mientras que los pastizales, los bosques naturales y los arbustos cubrían el resto. Estas cubiertas terrestres poseen buenas capacidades para retener la escorrentía superficial y apoyar la infiltración, causando un efecto opuesto a la urbanización. Esto puede explicar por qué el aumento del caudal es moderado a pesar del crecimiento significativo de la ciudad de Loja. Este hecho se analiza nuevamente en el escenario proyectado para 2025 (Tabla 12), que tendría un aumento del caudal basado en los datos históricos.

\section{CONCLUSIONES}

La pendiente, la elevación y la proximidad de las carreteras condicionaron el crecimiento urbano, por lo que hubo persistencia de las diferentes coberturas de tierra en el área de estudio. La mejor estimación del cambio en la cobertura de la tierra se encontró mediante regresión logística; sin embargo, las redes neuronales tuvieron un rendimiento similar.

Existe una relación directa entre el incremento de superficie urbana y la magnitud de los caudales de crecida a producirse por un evento extremo de precipitación. Las cuencas que experimentan mayor crecimiento de superficie urbana son las que presentan un mayor incremento en sus caudales de crecida, observándose una relación de tipo lineal. Si el porcentaje de área cubierta por un uso de suelo de tipo urbano es reducido en comparación al de las zonas ocupadas por vegetación en buenas condiciones, el incremento de los caudales de crecida será moderado.

El proceso de urbanización influye directamente en el ciclo hidrológico reduciendo la capacidad de infiltración, incrementando la magnitud de los caudales de crecida, es por esto que en la planificación urbana este hecho debe considerarse. 


\section{REFERENCIAS}

Carvajal K., Piedra R. (2007) Inventario Hidrológico de la Cuenca Superior del Río Zamora aplicando Sistemas de Información Geográfica (SIG). Tesis de grado. Universidad Nacional de Loja.

Chelsea Nagy, R., Graeme Lockaby, B., Kalin, L., Anderson, C. (2012) Effects of urbanization on stream hydrology and water quality: the Florida Gulf Coast. Hydrol. Process. 26, 2019-2030.

Chow V.T, R. Maidmentm, L. Mays. (1994) “Hidrología aplicada”. McGraw, Hill Bogotá.

Chuvieco E. (2010) Teledetección ambiental, Ariel ediciones, Madrid

Cruise, J.F., Laymon, C.A., Al-Hamdan, O.Z. (2010) Impact of 20 years of land-cover change on the hydrology of streams in the Southeastern United States1. JAWRA J. Am. Water Resour. Assoc. 46, 1159-1170.

Dingman, L. (2015), Physical Hydrology: Third Edition, Waveland Press, Long Grove, Illinois

Eastman, J.R. (2006) IDRISI Andes. Tutorial. Clark-Labs, Clark University, Worcester, MA.

Fletcher, T.D., H. Andrieu, P. Hamel. (2013) Understanding, management and modelling of urban hydrology and its consequences for receiving waters: A state of the art. Advances in Water Resources 51: 261-279

Google (2019) Imágen de la ciudad de Loja. Google Earth Pro. Fecha de consulta marzo de 2019

Hegerl, G.C., von Storch, H., Hasselmann, K., Santer, B.D., Cubasch, U., Jones, P.D., (1996) Detecting greenhouse-gas-induced climate change with an optimal fingerprint method. J. Clim. 9, 2281-2306.

Huang, J.-C., Lin, C.-C., Chan, S.-C., Lee, T.-Y., Hsu, S.-C., Lee, C.-T., Lin, J.-C. (2012) Stream discharge characteristics through urbanization gradient in Danshui River, Taiwan: perspectives from observation and simulation. Environ. Monit. Assess. 184, 5689-5703.

IGM (2018) Catálogo de datos del IGM. Instituto Geográfico Militar. Quito.

INAMHI (2019) Determinación de ecuaciones para el cálculo de intensidades máximas de precipitación. Versión 2. Instituto Nacional de Hidrología y Meteorología, Quito, Ecuador, 282 p.

Kleinbaum, D. G., Klein, M. (2014) Logistic Regression. A Self-Learning Text, Second Edition, Springer, N.Y. 513 p.

MAG (2019) Sistema de información pública agropecuaria, Ministerio de agricultura y ganadería del Ecuador. Quito

Municipio de Loja (2003) Actualización del catastro urbano de Loja. Municipio de Loja, Loja, Ecuador

Municipio de Loja (2014) Plan de desarrollo y ordenamiento territorial, actualización 2014-2022. Fase I. Municipio de Loja, Loja, Ecuador.

Oñate-Valdivieso, F., Sendra, J. (2010) Application of GIS and remote sensing techniques in generation of land use scenarios for hydrological modeling. Journal of Hydrology 395(3), 256 - 263

Oñate-Valdivieso, F., Sendra, J. (2014) Semidistributed Hydrological Model with Scarce Information: Application to a Large South American Binational Basin, J. Hydrol. Eng., 19(5), 1006-1014. 
Pijanowski, B. C., Brown D. G., Shellito B. A., Manik G. A. (2002) Using neural networks and GIS to forecast land use changes: a Land Transformation Model. Computers, Environment and Urban Systems. 26, 553-575.

Pontius, R., Shusas, E., McEachern (2004) Detecting important categorical land changes while accounting for persistence, Agriculture Ecosystems \& Environment, 101(2):251-268

Santer, B.D., Mears, C., Doutriaux, C., Caldwell, P., Gleckler, P.J., Wigley, T.M.L., Solomon, S., Gillett, N.P., Ivanova, D., Karl, T.R., Lanzante, J.R., Meehl, G.A., Stott, P.A., Taylor, K.E., Thorne, P.W., Wehner, M.F., Wentz, F.J. (2011) Separating signal and noise in atmospheric temperature changes: the importance of timescale. J. Geophys. Res. Atmos. 116, n/a-n/a.

United Nations. (2010) World urbanization prospects: the 2009 revision. New York: United Nations Department of Economic and Social Affairs (Population Division)

Zhang, X., Zwiers, F.W., Hegerl, G.C., Lambert, F.H., Gillett, N.P., Solomon, S., Stott, P.A., Nozawa, T. (2007). Detection of human influence on twentieth-century precipitation trends. Nature 448, 461465.

Zheng, J., Yu, X., Deng, W., Wang, H., Wang, Y. (2012). Sensitivity of land-use change to streamflow in Chaobai River Basin. J. Hydrol. Eng. 18, 457-464. 